\title{
Preparation of Site Specific Atom Probe Tips using Focused Ion Beam Technology
}

D. McGrouther ${ }^{*}$, W. McKenzie*, D. Saxey ${ }^{* *}$, J.M. Cairney ${ }^{* *}$, R. Marceau**, S.P. Ringer ${ }^{* *}$ and P.R. Munroe*

* NANO@ University of New South Wales, NSW 2052, Australia

**NANO@ University of Sydney, NSW 2006, Australia

Atom probe (AP) tomography is finding increasing application in materials analysis. This technique provides high-resolution structural and chemical data, but the requirements for specimen

preparation are exacting; viz posts where the radius undergoing analysis is less than $50 \mathrm{~nm}$ in diameter. Historically, it has been metals that have been predominantly examined, where tip preparation has been performed via electro-polishing techniques. This is reasonably straightforward and rapid, but provides only limited control over the location of the tip relative to the bulk. However, near future development in AP instrumentation, with respect to pulsed laser technology, will provide conditions for analysis of materials with lower electrical conductivity, and will include applications in multi-layered thin films or semiconducting devices. For these materials alternate methods of specimen preparation are required. Focused ion beam (FIB) instruments are well known in the ability to mill in precise locations of a specimen, and have been used to prepare successfully AP specimens from metallic specimens [1,2]. Subsequently, this method has been extended to more site-specific locations and more complex materials [3], typically through the preparation of posts from areas where thin lamellae, prepared as transmission electron microscope (TEM) specimens, are used as a starting point. In this paper we demonstrate a rapid methodology for the preparation of AP posts that allows, for example, flexibility in the orientation of any material interfaces with respect to the longitudinal axis of the post or the ability to generate both AP specimens and TEM specimens in the same post.

In this example, AP post preparation is performed in a dual beam FIB, but may be easily adapted to be performed in a single beam FIB. The technique requires no pre-preparation. First, the area of interest is identified and, if required, a protective strip of Pt up to $200 \mu \mathrm{m}$ in length, and typically $\sim 5$ $\mu \mathrm{m}$ in width and $\sim 1 \mu \mathrm{m}$ in thickness is deposited over the area of interest (In the specific example shown Pt deposition was not performed). The specimen is tilted $\sim 7^{\circ}$ with respect to the electron column, which translates to $45^{\circ}$ relative to ion beam. Using a $7 \mathrm{nA}$ ion beam, a trench, up to 200 $\mu \mathrm{m}$ in length is milled along the length of the region of interest $\sim 0.5 \mu \mathrm{m}$ wide, typically to a depth of $5 \mu \mathrm{m}$. The stage is rotated $180^{\circ}$ and an identical cut is performed parallel to the original mill, such that the two cuts are about $5 \mu \mathrm{m}$ apart (fig. 1). The specimen is tilted back to a $52^{\circ}$ orientation with respect to the electron beam, that is, so the specimen surface is normal to the ion beam. Regular cross-sectional mills are used to free each end of the bar. This exposes the cuts on each end of the bar (fig. 2). A cleaning cross-section may be needed to remove any re-deposition. The result is a post of material lying free in the trench. An ex-situ nanomanipulator with a metal needle is used to nudge the post in the trench ensuring it is free. Then, a glass needle is used to pick up the post and land it on the edge of a half-copper slot grid. (This procedure could also be performed with an in-situ nanomanipulator). The slot grid and post are returned to the FIB and Pt is used to weld the post to the gird (fig. 3). The post is oriented such that its longitudinal axis is normal to the ion beam 
and a series in annular mills, in a manner similar to described elsewhere [3], is made to reduce the diameter of the post to one suitable for AP analysis (fig. 4). The post can be extracted from thin film structures such that any interfaces may lie along the longitudinal axis of the post. Further variations of this method include the possibility of performing final mills such that both an AP tip, and an electron transparent section, suitable for TEM analysis, can be prepared from the same post. Thus, specimens can be examined in the TEM prior to AP analysis. Typical preparation time is from two to four hours. This approach is applicable to a wide range of materials including metals, semiconducting devices and thin film multilayers. If required, the post may also be readily returned to the FIB for re-sharpening of the tip.

\section{References}

[1] D.J. Larson et al., Ultramicroscopy 75 (1998) 147.

[2] D.J. Larson et al., Ultramicroscopy 79 (1999) 287.

[3] M.K. Miller et al., Ultramicroscopy 102 (2005) 287.

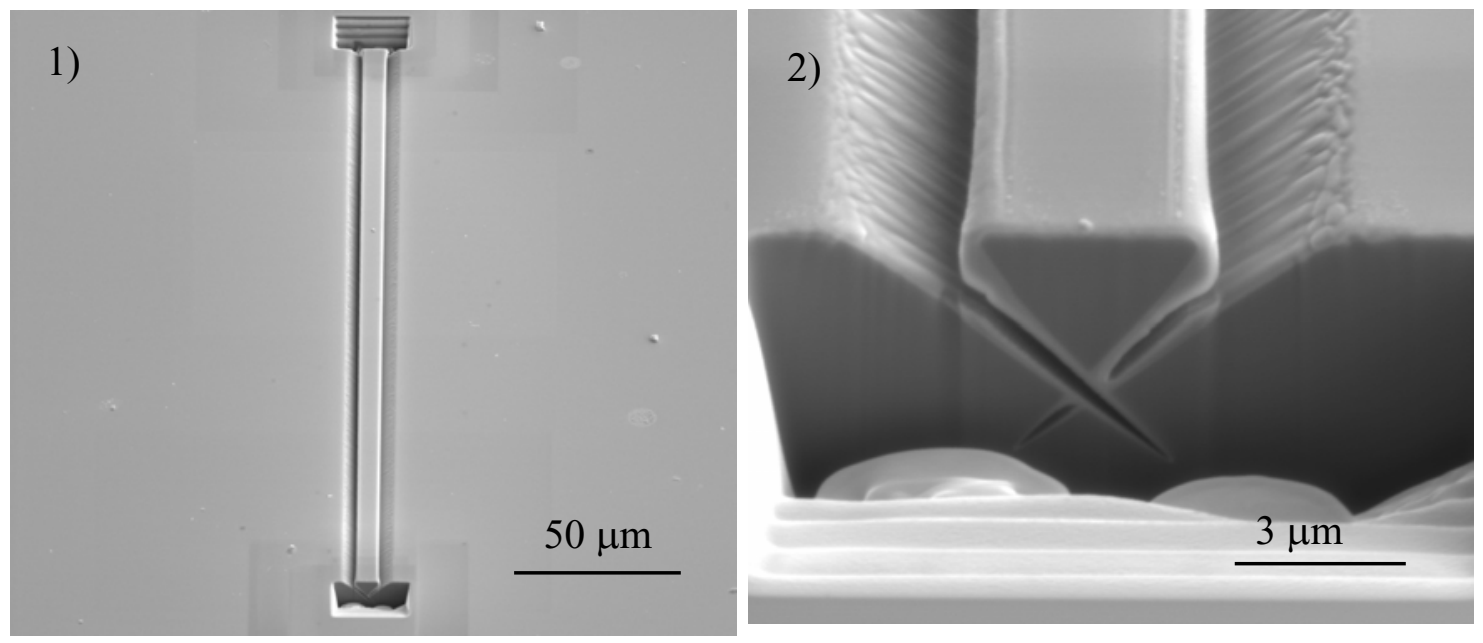

Fig. 1: Secondary electron image of initial parallel cuts at the first stage of post preparation. Fig. 2: Secondary electron image of one end of the post freed by cross-sectional milling
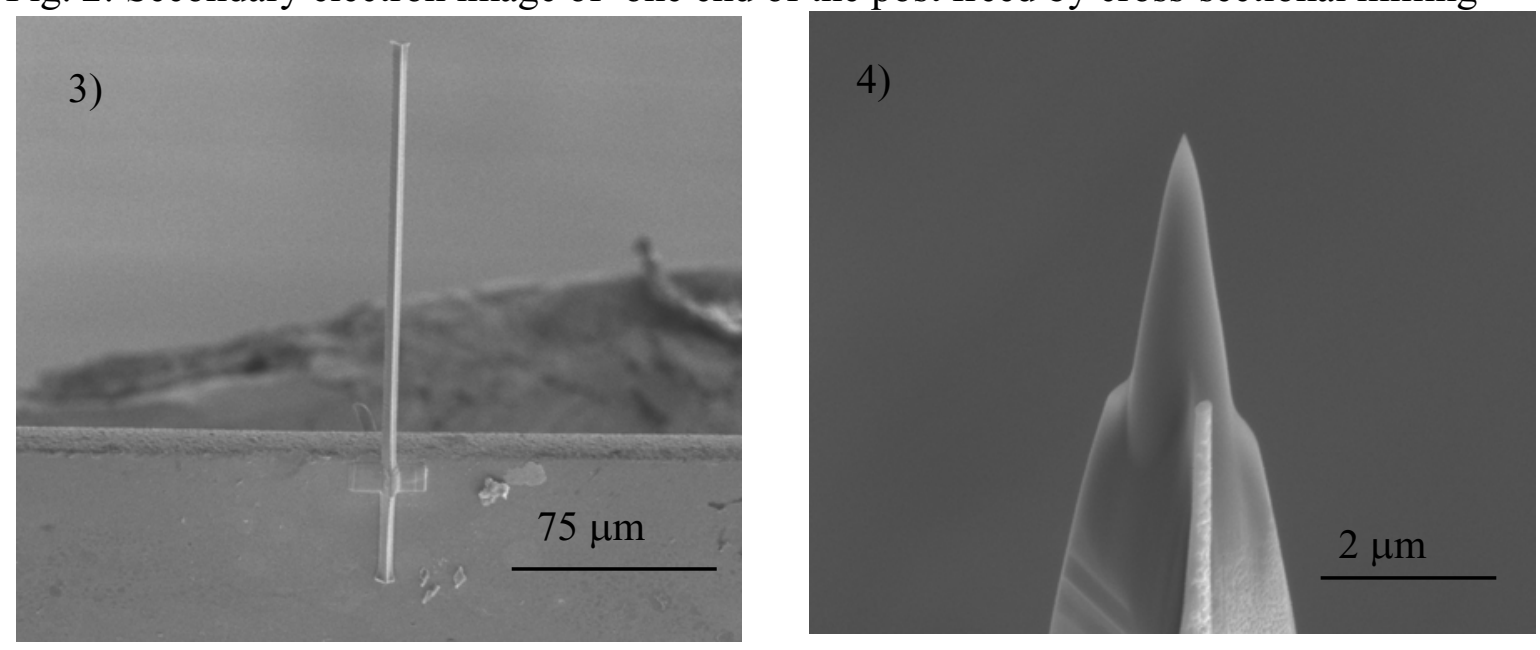

Fig. 1: Secondary electron image of the post welded with $\mathrm{Pt}$ to a $\mathrm{Cu}$ slot grid Fig. 2: Secondary electron image of tip following final milling 